\title{
Fetuin-A modulates lipid mobilization in bovine adipose tissue by enhancing lipogenic activity of adipocytes
}

\author{
Clarissa Strieder-Barboza* and G. Andres Contreras† \\ Department of Large Animal Clinical Sciences, College of Veterinary Medicine, Michigan State University, East Lansing 48824
}

\begin{abstract}
Fetuin-A (FetA) is an adipokine and free fatty acid (FFA) carrier linked to adipose tissue (AT) function in monogastrics and ruminants. In dairy cows, plasma and AT FetA decrease after parturition, coinciding with reduced lipogenesis and increased lipolysis. In monogastrics, FetA enhances lipogenesis, but its role on lipid mobilization of ruminants is unclear. We hypothesized that FetA modulates lipid mobilization in bovine AT by enhancing the lipogenic activity of adipocytes. Our objective was to determine the effects of FetA on lipogenesis and lipolysis in cultured primary adipocytes from dairy cows. Preadipocytes from the tailhead subcutaneous AT depot were induced to differentiate in a 7-d coculture in vitro model. The effects of FetA on lipolytic responses of adipocytes were evaluated after a 2-h $\beta$-adrenergic stimulation with $1 \mu M$ isoproterenol (ISO) alone or combined with $0.1 \mathrm{mg} / \mathrm{mL}$ of FetA (FetA+ISO), and in cells treated with medium alone $(\mathrm{CON})$ or with $0.1 \mathrm{mg} / \mathrm{mL}$ of FetA (FetA). Lipogenic responses of adipocytes treated with CON or FetA from d 5 to 7 of differentiation were assessed by fatty acid (FA) uptake quantification and triacylglycerol (TAG) accumulation, and the gene and protein expression of lipogenic markers. Bovine adipocytes abundantly expressed FetA gene and protein and secreted $48 \pm 3.5$ ng/DNA relative fluorescence units (RFU). Adrenergic stimulation with ISO increased lipolysis compared with $\mathrm{CON}$, as reflected in the release of glycerol $(0.12 \pm 0.04$ vs. $0.04 \pm 0.02 \mathrm{nM} / \mathrm{DNA} \mathrm{RFU})$ and FFA ( $15 \pm 13$ vs. $6.2 \pm 2.4 \mathrm{n} M / \mathrm{DNA}$ RFU). Lipolysis induced by ISO was attenuated by the addition of FetA (FetA+ISO) as reflected by lower glycerol $(0.06 \pm 0.04 \mathrm{n} M / \mathrm{DNA} R F \mathrm{R})$ and FFA $(5.7 \pm 2.7 \mathrm{n} M / \mathrm{DNA} \mathrm{RFU})$ release compared with ISO alone. Compared with CON, FetA enhanced lipogenic responses as demonstrated by higher FA
\end{abstract}

\footnotetext{
Received October 8, 2018.

Accepted January 6, 2019.

*Current affiliation: Department of Surgery, Medical School, University of Michigan, Ann Arbor 48109.

†Corresponding author: contre28@msu.edu
}

uptake and increased accumulation of TAG. Exposure to FetA upregulated 1-acylglycerol-3-phosphate acyltransferase-2 (AGPAT2) gene expression and protein content, as well as its activity. Adipocytes exposed to FetA increased the secretion of the metabolite of AGPAT2, phosphatidic acid. In conclusion, FetA attenuates lipolytic responses and enhances lipogenesis in bovine adipocytes. The upregulation of the ratelimiting lipogenic enzyme AGPAT2 by FetA suggests a potential pathway by which this adipokine promotes TAG synthesis in adipocytes. These findings suggest that FetA is a potential target for lipid mobilization modulation in AT of dairy cows.

Key words: adipocyte, dairy cow, lipogenesis, lipolysis

\section{INTRODUCTION}

Increased lipid mobilization in adipose tissues (AT) is the major metabolic adaptation to negative energy balance in periparturient dairy cows. During lipolysis, one molecule of triacylglycerol (TAG) is broken down into 3 free fatty acid (FFA) molecules and 1 molecule of glycerol through the activity of adipose triglyceride lipase, hormone-sensitive lipase, and monoglyceride lipase. Released FFA are either re-esterified into TAG within the adipocytes or used as an energy source elsewhere in the body (Vernon and Pond, 1997). Lipogenesis involves the biosynthesis of TAG from fatty acids (FA) either synthesized from FFA released from blood TAG or de novo within the adipocytes. Glycerol phosphate pathway is the major pathway for de novo synthesis of TAG by adipocytes, which consists in a stepwise addition of fatty acyl groups catalyzed by distinct enzymes: glycerol-3-phosphate acyltransferase (GPAT), 1-acylglycerol-3-phosphate acyltransferase (AGPAT), lipins (phosphatidate phosphatases), and diacylglycerol acyltransferase (DGAT) to finally form TAG (Takeuchi and Reue, 2009). Adipocyte TAG reserves serve as the main source of energy to offset negative energy balance in periparturient cows (McNamara, 1995).

Around parturition, lipolysis is enhanced and lipid synthesis is reduced in adipocytes by physiological factors such as decreased plasma insulin and glucose 
concentrations, impaired insulin sensitivity in AT and other peripheral tissues, and increased concentrations of catecholamines, growth hormone, and glucocorticoids (Bauman and Currie, 1980). Also, a decrease in the transcription and activity of key lipogenic enzymes limits the ability of adipocytes to synthesize FA and TAG thus enhancing FFA release from AT (McNamara, 1995). The relative balance between lipolysis and lipogenesis in AT controls the release of FFA from adipocytes and is critical to ensure a successful transition from gestation to lactation in dairy cows. Dysregulated lipid mobilization leads to a massive release of FFA into circulation that, coupled with an excessive accumulation of lipids in the liver and exacerbated pro-inflammatory responses, predisposes periparturient dairy cows to health events (Vernon and Houseknecht, 2000; Contreras et al., 2018). Currently, a major challenge in the field of periparturient dairy cow management is the adequate regulation of AT lipolysis and lipogenesis rates to ensure that released FFA are fully metabolized for energy needs, thus avoiding the detrimental lipid accumulation in blood and tissues.

We recently determined the dynamics of a novel adipokine, fetuin-A (FetA; $\alpha$-2-Heremans-Schmid glycoprotein; encoded by $A H S G$ gene), in serum and AT of dairy cows during the periparturient period (Strieder-Barboza et al., 2018). Our results indicated that FetA is a negative acute-phase adipokine in the subcutaneous AT and that its plasma concentrations were negatively associated with markers of AT lipid mobilization including BHB and BCS loss. In the same study, we reported FetA gene and protein expression in bovine adipocytes, suggesting a potential autocrine role for this adipokine (Strieder-Barboza et al., 2018). Even though FetA was shown to be expressed by bovine $\mathrm{AT}$, its secretion by primary adipocytes has not yet been reported. In humans and mice, FetA secretion by visceral and subcutaneous AT was augmented during obesity and in animals fed a high-fat diet (Jialal et al., 2015; Pérez-Sotelo et al., 2017). In monogastric adipogenic models, this effect is probably related to the lipogenic properties of FetA as this protein is a carrier of lipids in plasma and facilitates the incorporation of exogenous FA into intracellular TAG (Kumbla et al., 1989; Cayatte et al., 1990). Because low FetA in plasma and AT coincided with low lipogenesis and high lipolysis rates in periparturient cows, and given the fact that FetA was associated with pro-lipogenic states in nonruminant species, we hypothesized that FetA modulates lipid mobilization by enhancing lipogenic activity in bovine adipocytes. Our objectives were to determine the effect of FetA on lipogenesis and lipolysis, and to identify potential mechanisms by which this adipokine modulates lipid mobilization of bovine adipocytes.

\section{MATERIALS AND METHODS}

All animal procedures were approved by the Michigan State University Animal Care and Use Committee.

\section{Tissue Collection and Processing}

Subcutaneous AT from the tailhead depot from 7 nonlactating, nongestating, multiparous Holstein dairy cows was collected in Krebs-Ringer modified buffer supplemented with HEPES $10 \mathrm{~m} M(\mathrm{pH}=7.4)$ at a local slaughterhouse as previously described (StriederBarboza et al., 2018). In brief, AT (1 g) was digested with $5 \mathrm{~mL}$ of collagenase type II solution $(2 \mathrm{mg} / \mathrm{mL}$; Worthington Biochemical, Lakewood, NJ) and then centrifuged $\left(800 \times g, 10 \mathrm{~min}, 4^{\circ} \mathrm{C}\right)$ to separate the primary adipocytes from the stromal vascular fraction (SVF). Primary mature adipocytes were washed in $5 \mathrm{~mL}$ of Krebs-Ringer modified buffer with 4\% BSA (Millipore-Sigma, Sigma-Aldrich, St. Louis, MO), centrifuged, and then retained for use in transwell inserts for inductions using a coculture protocol (Thelen et al., 2018). The SVF was then sequentially filtered through 100- and $40-\mu \mathrm{m}$ cell strainers (Falcon, Corning, Corning, NY) and centrifuged. The resulting cell pellet was resuspended and incubated in erythrocyte lysis buffer. After another centrifugation, resultant cells were resuspended in basal preadipocyte medium containing Dulbecco's modified Eagle's medium: F12 (Corning), 10\% fetal bovine serum (Corning), $2 \mathrm{mmol} / \mathrm{L}$ of L-glutamine (Corning), 1\% (vol/vol) antibiotic-antimycotic (Corning), $44.05 \mathrm{mmol} / \mathrm{L}$ of sodium bicarbonate (SigmaAldrich, St. Louis, MO), $100 \mu \mathrm{mol} / \mathrm{L}$ of ascorbic acid (Sigma-Aldrich), $33 \mu \mathrm{mol} / \mathrm{L}$ of biotin (Sigma-Aldrich), $17 \mu \mathrm{mol} / \mathrm{L}$ of pantothenate (Sigma-Aldrich), and 20 $\mathrm{mmol} / \mathrm{L}$ of HEPES (Corning) and incubated at $37^{\circ} \mathrm{C}$ in a humidified atmosphere with $5 \% \mathrm{CO}_{2}$ with medium replacement every $48 \mathrm{~h}$ (Strieder-Barboza et al., 2018). Preadipocytes were obtained by outgrowth of plasticadherent cells from the SVF cells after 2 serial passages in culture flasks (Corning).

\section{Cell Induction and Differentiation}

Expanded preadipocytes populations were seeded in 6-, 12-, or 24-well plates (Corning) and allowed to proliferate to confluency. Preadipocytes were induced to differentiate after $48 \mathrm{~h}$ at $100 \%$ confluency (d 0) using a coculture model (Thelen et al., 2018). Briefly, 900 mature adipocytes $/ \mathrm{cm}^{2}$ were placed in $0.4-\mu \mathrm{m}$ transwell inserts (Greiner Bio-One, Kremsmünster, Austria) over the attached preadipocytes for the first $5 \mathrm{~d}$ of differentiation. For differentiation induction, cells were incubated with basal medium supplemented 
with $10 \%$ fetal bovine serum, $5 \mu \mathrm{mol} / \mathrm{L}$ of troglitazone (Cayman Chemical, Ann Arbor, MI), $0.5 \mathrm{mmol} / \mathrm{L}$ of 2 isobutyl-1-methylaxanthine (AdipoGen Life Sciences, San Diego, CA) and the following reagents from Sigma Aldrich: $5 \mu \mathrm{g} / \mathrm{mL}$ of insulin, $10 \mathrm{~m} M$ acetate, and 1 $\mu \mathrm{mol} / \mathrm{L}$ of dexamethasone for $7 \mathrm{~d}$. The 2 isobutyl1-methylaxanthine and dexamethasone were used only during the first $48 \mathrm{~h}$ of induction, and medium changes were performed every $48 \mathrm{~h}$. Adipocyte lipid accumulation was assessed quantitatively in triplicates per experimental unit using a 96-well plate for the AdipoRed assay (Lonza, Allendale, NJ) and a Synergy H1 Microplate Reader (Biotek, Winooski, VT). Fetuin-A dose of $0.1 \mathrm{mg} / \mathrm{mL}$ was used for all metabolic assays and was established based on previous studies with adipocytes (Heinrichsdorff and Olefsky, 2012; Pal et al., 2012), as well as on a dose response assay $(0,50,100,250,500$, and $1,000 \mu \mathrm{g} / \mathrm{mL}$ of FetA) having the gene expression of lipogenic and inflammatory genes as the outcome.

\section{Lipolysis Assay}

Induction of lipolysis was performed using the nonselective $\beta$-adrenergic receptor agonist isoproterenol (ISO; Sigma-Aldrich). Briefly, cultured adipocytes (n $=6$ ) were removed from 6 -well plates (Corning) using trypsin (Thermo Fisher Scientific, Waltham, MA), seeded in triplicate at $1 \times 10^{5}$ cells/well in black wellclear bottom 96-well plates (Nunc, Roskilde, Denmark), and allowed to attach for $4 \mathrm{~h}$ at $37^{\circ} \mathrm{C}$ in a humidified atmosphere with $5 \% \mathrm{CO}_{2}$ (Supplemental Figure S1; https://doi.org/10.3168/jds.2018-15808). Adipocytes were then starved for $4 \mathrm{~h}$ with serum-free-Dulbecco's modified Eagle medium: F12 supplemented with 0.1\% FA free BSA (Millipore-Sigma). Lipolytic responses of adipocytes were evaluated after $2 \mathrm{~h}$ of incubation at $37^{\circ} \mathrm{C}$ with Krebs-Ringer bicarbonate HEPES buffer (pH 7.4) containing 3\% FA free BSA (Millipore-Sigma) supplemented with $1 \mu M$ ISO alone, $0.1 \mathrm{mg} / \mathrm{mL}$ of FetA alone (FetA; catalog no. 341506, Millipore, Darmstadt, Germany), or the combination of $0.1 \mathrm{mg} / \mathrm{mL}$ of FetA and $1 \mu M$ isoproterenol (ISO+FetA). Basal lipolysis was determined without the addition of any reagent $(\mathbf{C O N})$. All reagents were prepared fresh on the day of the experiment. Lipolytic responses were assessed by the concentrations of glycerol (catalog no. MAK1171KT, Millipore-Sigma) and FFA (HR Series NEFAHR (2), Fujifilm Wako Diagnostics U.S.A., Mountain View, $\mathrm{CA}$ ) released in the culture medium during the 2-h assay. To evaluate whether FetA is secreted by bovine adipocytes, we analyzed FetA concentrations in $\mathrm{CON}$ cell culture medium. The FetA concentrations were determined by ELISA following the manufacturer's guidelines (cow AHSG/fetuin-A, catalog no. LS-F6106,
LSBio, Seattle, WA) as reported previously (StriederBarboza et al., 2018). All conditions were performed in triplicate. Statistical analysis was performed using the average glycerol and FFA concentrations of the triplicates. Concentrations of glycerol (nM), FFA (nM), and FetA $(\mathrm{ng} / \mathrm{mL})$ were adjusted by the postfunctional analysis of cellular DNA content as determined by relative fluorescence units (RFU) using CyQUANT (Life Technologies, Carlsbad, CA).

\section{Fatty Acid Uptake Assay}

Fatty acid uptake analysis was performed using the kinetic QBT Fatty Acid Uptake Assay (Molecular Devices, Sunnyvale, CA). Cultured adipocytes (n $=6$ ) were detached by trypsinization and seeded as described for the lipolysis assay. Viability after trypsin detachment was assessed by Trypan blue exclusion. Adipocytes were then serum-starved overnight and pre-incubated with $0.1 \mathrm{mg} / \mathrm{mL}$ of FetA, $10 \mathrm{n} M$ insulin (insulin, positive control; Sigma-Aldrich) or serum-free basal preadipocyte medium (CON; basal FFA uptake) during $30 \mathrm{~min}$ at $37^{\circ} \mathrm{C}$ in a humidified atmosphere with $5 \% \mathrm{CO}_{2}$. Next, the reagent of the QBT Fatty Acid Uptake assay containing a fluorescent-labeled FA analog was added to the cells $(100 \mu \mathrm{L} /$ well $)$. Kinetic uptake was measured every $20 \mathrm{~s}$ for $1 \mathrm{~h}$ using a Synergy H1 Microplate Reader (Biotek). Triplicate values per sample were averaged, subtracted from blank, and divided by basal values $(\mathrm{CON})$ to calculate the area under the curve for each treatment. Values are expressed as fold change over CON.

\section{Triacylglycerol Accumulation}

Intracellular TAG accumulation in adipocytes was assessed by Adipogenesis Assay Reagent (AdipoRed, Lonza) and the lipid droplet staining HCS LipidTox (Life Technologies) analyzed through confocal microscopy imaging. Cultured adipocytes were incubated with basal adipocyte differentiation medium supplemented with $0.1 \mathrm{mg} / \mathrm{mL}$ of FetA for $48 \mathrm{~h}$ (d 5 to 7 of the differentiation protocol). Basal TAG accumulation was determined in adipocytes incubated with basal differentiation medium without addition of FetA (CON).

Lipid Droplet Staining and Confocal Microscopy Imaging. After 48 h-incubation with or without FetA, adipocytes $(n=8)$ were seeded in duplicates/ treatment per cow in a glass bottom 24-well plate (Corning) at a concentration of 20,000 cells $/ \mathrm{cm}^{2}$. Then DAPI (NucBlue, Life Technologies) and HCS LipidTox with Alexa Fluor 594 (Life Technologies) were used to visualize adipocytes' nuclei and intracellular TAG, respectively. These dyes were used according to 
the manufacturer's protocols. Briefly, adipocytes were fixed with $4 \%$ paraformaldehyde (Electron Microscopy Sciences, Hatfield, PA) in PBS $(1 \times$ PBS) for $20 \mathrm{~min}$ at room temperature. Two hundred fifty microliters of HCS LipidTOX neutral lipid stain 1:200 in 1× PBS was added to each well (Corning) and incubated at room temperature for $30 \mathrm{~min}$. The buffer was removed and $200 \mu \mathrm{L}$ of DAPI (300 $\mathrm{n} M$ in $1 \times$ PBS) was added to each well and incubated at room temperature for 5 min. Images were acquired using an Olympus FluoView 1000 Confocal Laser Scanning Microscope (Olympus America Inc., Center Valley, PA) configured on an IX81 inverted microscope and FV10-ASW software (version 4.2.3.6) using a PLAPON $60 \times / 1.42$ oil objective. Alex Fluor 594 fluorescence (577 nm excitation/609 $\mathrm{nm}$ emission) was excited with the $543 \mathrm{~nm}$ HeNe gas laser, detected using a BP 560IF emission filter, and displayed in red color. The DAPI fluorescence (358 nm excitation/461 nm emission) was excited using the 405 $\mathrm{nm}$ diode laser, detected using a 430 to $470 \mathrm{~nm}$ band pass emission filter, and displayed in blue color. Images were obtained using sequential single confocal XY scan mode. Control images for TAG accumulation included preadipocytes and nonstained adipocytes. Relative fluorescence intensity of intracellular TAG per cell was determined by ImageJ (https://imagej.net/Welcome) software and calibrated by cell number.

Adipogenesis Assay Reagent. AdipoRed is a solution of Nile red, a hydrophilic stain, that fluoresces when partitioned into lipid droplets of differentiated adipocytes. Briefly, adipocytes $(\mathrm{n}=5)$ in triplicates/ treatment per cow in 24-well plates (Corning) were washed once with $1 \times$ PBS, incubated with AdipoRed assay reagent for $20 \mathrm{~min}$ at room temperature, and then analyzed for fluorescence intensity (excitation/emission $=485 / 572 \mathrm{~nm}$ ) using a Synergy H1 Microplate Reader (Biotek). Preadipocytes from each experimental unit on d 0 served as negative controls. Relative fluorescence units of adipocytes were calibrated by their correspondent preadipocyte RFU and are presented as fold change over preadipocyte lipid accumulation.

\section{Gene Expression Analysis of Adipogenesis and Lipogenesis Markers}

After treating adipocytes $(\mathrm{n}=5)$ for $48 \mathrm{~h}$ with or without $0.1 \mathrm{mg} / \mathrm{mL}$ of FetA, culture medium was removed, and cells were rinsed twice with ice-cold $1 \times$ PBS. Adipocytes' RNA was extracted using Promega simplyRNA Cells Kits (catalog no. AS1390, Promega, Madison, WI) in the Maxwell RSC Instrument (Promega) as described previously (Strieder-Barboza et al., 2018). This protocol started with the addition of 100 $\mu \mathrm{L}$ of 1-thioglycerol/homogenization solution to each of the 2-wells/treatment per cow of a 12 -well plate with adipocytes and then transferred to a microfuge tube. Next, $200 \mu \mathrm{L}$ of lysis buffer was added and homogenate was vortexed and then placed in Maxwell RSC Cartridges, which were previously loaded with 10 $\mu \mathrm{L}$ of DNase I. Purity, concentration, and integrity of AT and cells' RNA were evaluated using an Agilent Bioanalyzer 2100 (Agilent Technologies, Santa Clara, $\mathrm{CA}$ ). All samples had an RNA integrity number $\geq 6$. Conversion to cDNA was performed using the Applied Biosystems High Capacity cDNA Archive Kit (Applied Biosystems, Foster City, CA). Transcriptional studies were performed on the cDNA samples using quantitative PCR reactions on QuantStudio 7 Flex (Applied Biosystems). All qPCR reactions were performed in duplicates, and no-template controls were included on each plate for each TaqMan gene expression assay.

Gene expression data of 5 endogenous control genes (B2M, EIF3K, GAPDH, RPLP0, and RPS9) were analyzed using qBase+ analysis software, which calculates the stability of endogenous control genes (M-value). Following qBase+ analysis of gene expression data, endogenous control genes EIF3K and RPS9 were ranked best. The $\mathrm{Cq}$ values of the target genes (ADIPOQ, AGPAT2, AHSG, CD36, CEBPB, DGAT1, DGAT2, FABP 4, FATP1, GPAT1, GPAT2, LPIN1, and PPARG) were converted to normalized relative gene expression as described previously (Hellemans et al., 2007; Contreras et al., 2017a). The quantitative PCR assays were conducted in duplicates with TaqMan gene expression assays from Applied Biosystems, except FetA (AHSG), which was provided by Integrated DNA Technologies (IDT, Coralville, IA) as described previously (StriederBarboza et al., 2018; Supplemental Table S1; https:// doi.org/10.3168/jds.2018-15808).

\section{Western Blotting}

Western blots were performed as previously described (Strieder-Barboza et al., 2018). Protein from cultured adipocytes $(\mathrm{n}=8)$ was extracted from 3 wells/treatment per cow of a 12-well plate using ice-cold RIPA buffer (Teknova, Hollister, CA) containing protease (Roche, San Francisco, CA) and phosphatase (Thermo Fisher Scientific) inhibitors. Estimation of protein content was carried out using the Pierce BCA Protein Assay Kit (Thermo Fisher Scientific). Fetuin-A content was analyzed using reducing conditions. Briefly, samples were added to a reducing buffer containing 10 $\mathrm{mmol} / \mathrm{L}$ of dithiothreitol and $5 \% \beta$-mercaptoethanol and denatured at $95^{\circ} \mathrm{C}$ for $4 \mathrm{~min}$. Twenty micrograms of protein was separated by electrophoresis on a 4 to 
$20 \%$ SDS-PAGE gel and transferred to a polyvinylidene difluoride membrane. The membrane was blocked in Tris-buffered saline solution with $0.01 \%$ Tween-20 (TBST) and 5\% fat-free milk. Membranes were incubated with a conjugated polyclonal rabbit anti-bovine AGPAT2 antibody (C-terminal region catalog no. ARP44636_P050, Aviva Systems Biology Corporation, San Diego, CA) in $1 \mu \mathrm{g} / \mathrm{mL}$ concentration in TBST-5\% milk for $16 \mathrm{~h}$ at $8^{\circ} \mathrm{C}$. $\alpha \beta$-Tubulin $(1: 1,000$; catalog no. 2148; Cell Signaling Technology, Danvers, MA) served as loading control for adipocyte protein. Membranes were then exposed to horseradish peroxidase substrate (catalog no. WBLUR0100, Millipore, Darmstadt, Germany) and visualized by chemiluminescence using the ChemiDoc Touch Imaging System (Bio-Rad, Hercules, CA). Band densitometry was determined using the Image Lab software (Bio-Rad). Values of AGPAT2 protein content are expressed as means of relative band density using $\alpha \beta$-tubulin band intensity values as calibrator.

\section{Phosphatidic Acid Analysis}

Phosphatidic acid (PA) concentrations in adipocytes were measured using the enzymatic fluorometric PicoProbe Phosphatidic Acid Assay Kit (catalog no. K748, BioVision Inc., Milpitas, CA) following the manufacturer's instructions. Briefly, after treating adipocytes $(\mathrm{n}=5)$ with or without $0.1 \mathrm{mg} / \mathrm{mL}$ of FetA for $48 \mathrm{~h}$, culture medium was removed, and cells from 3 wells/treatment/cow of a 12 -well plate were rinsed twice with ice-cold $1 \times$ PBS, scraped from cell culture plates, and collected in $1 \times$ PBS. For lipid extraction, 1 $\times 10^{6}$ adipocytes were mixed with $1 \mathrm{~mL}$ of assay buffer (BioVision) and sonicated using an ultrasonic liquid processor (Misonix, Farmingdale, NY) for 2 min at $4^{\circ} \mathrm{C}$. Protein content was estimated at this point using a BCA Protein Assay Kit (Thermo Fisher Scientific). Following this, $3.75 \mathrm{~mL}$ of chloroform/methanol/12 N $\mathrm{HCl}$ (2: 4: $0.1 \mathrm{vol} / \mathrm{vol}$; Sigma-Aldrich) was added to the cell homogenate and mixed thoroughly. Then, 1.25 $\mathrm{mL}$ of chloroform was added to the solution, vortexed for $30 \mathrm{~s}$, and added to $1.25 \mathrm{~mL}$ of $1 \mathrm{M} \mathrm{NaCl}$ (SigmaAldrich). Followed by centrifugation, the lower organic layer containing solubilized lipids was collected and transferred to a glass tube. Chloroform was evaporated in a vacuum concentrator (Savant SPD121P, Thermo Fisher) for $2 \mathrm{~h}$ at $40^{\circ} \mathrm{C}$. Before performing the analysis, samples were solubilized in $50 \mu \mathrm{L}$ of $5 \%$ Triton X-100 solution. For PA measurement, $10 \mu \mathrm{L}$ of sample was added to a 96 -well plate and incubated for $1 \mathrm{~h}$ at $45^{\circ} \mathrm{C}$ with a compound that hydrolyzes PA. Samples were then incubated for $30 \mathrm{~min}$ at $37^{\circ} \mathrm{C}$ in the presence of a developer and enzyme mix that converts a nonfluores- cent probe to a fluorescent product $(\mathrm{Ex} / \mathrm{Em}=535 / 587$ $\mathrm{nm}$ ) that can be quantified (Synergy H1 Microplate Reader, Biotek). Concentrations of PA were calculated using the manufacturer's PA standard (BioVision) and L-A-phosphatidic acid sodium (P9511, Sigma-Aldrich). Phosphatidic acid values were obtained by subtracting the background RFU from each sample and then calibrated by the total protein concentration (nmol of PA per ng of protein).

\section{Statistical Analysis}

Data were analyzed using JMP Statistical Software (SAS Institute Inc., Cary, NC). Normality of the variables was checked using the Kolmogorov-Smirnov test $(P<0.05)$. Nonnormally distributed variables (FFA concentrations in lipolysis assay, FA uptake assay, adipocytes AGPAT2 protein content) were natural logarithm transformed. One-way ANOVA pairwise comparisons were performed using the Tukey's post hoc test. Mean differences were considered significant when $P \leq 0.05$ and tendencies when $P<0.10$. Average values throughout the text and figures are showed as mean \pm SEM.

\section{RESULTS}

\section{Bovine Adipocytes Abundantly Secrete Fetuin-A}

The capacity of primary bovine adipocytes to express and secrete FetA into the medium was assessed. We determined that these cells secrete $48 \pm 3.5 \mathrm{ng}$ of FetA/ DNA RFU per 2-h incubation in serum-free basal medium and express $A H S G$ and FetA protein at a similar level as those observed in AT of periparturient cows $(P>0.05$; Supplemental Figure S2; https://doi.org/10 $.3168 /$ jds.2018-15808).

\section{Fetuin-A Attenuates Lipolytic Responses in Bovine Adipocytes}

After assessing the ability of bovine adipocytes to secrete FetA, we evaluated the effect of FetA on lipolytic responses of bovine adipocytes. $\beta$-Adrenergic stimulation with ISO for $2 \mathrm{~h}$ increased the release of glycerol $(0.12 \pm 0.04$ vs. $0.04 \pm 0.02 \mathrm{n} M / \mathrm{DNA} \mathrm{RFU}$, $P=0.003)$ and FFA $(15 \pm 13$ vs. $6.2 \pm 2.4 \mathrm{n} M / \mathrm{DNA}$ RFU, $P=0.04$ ) compared with CON (Figure 1). Remarkably, ISO-induced lipolysis was attenuated by FetA (FetA+ISO) as reflected in lower glycerol (0.06 $\pm 0.04 \mathrm{n} M / \mathrm{DNA}$ RFU, $P=0.02)$ and FFA $(5.7 \pm 2.7$ $\mathrm{n} M / \mathrm{DNA}$ RFU, $P=0.01)$ release compared with ISO alone (Figure 1). 

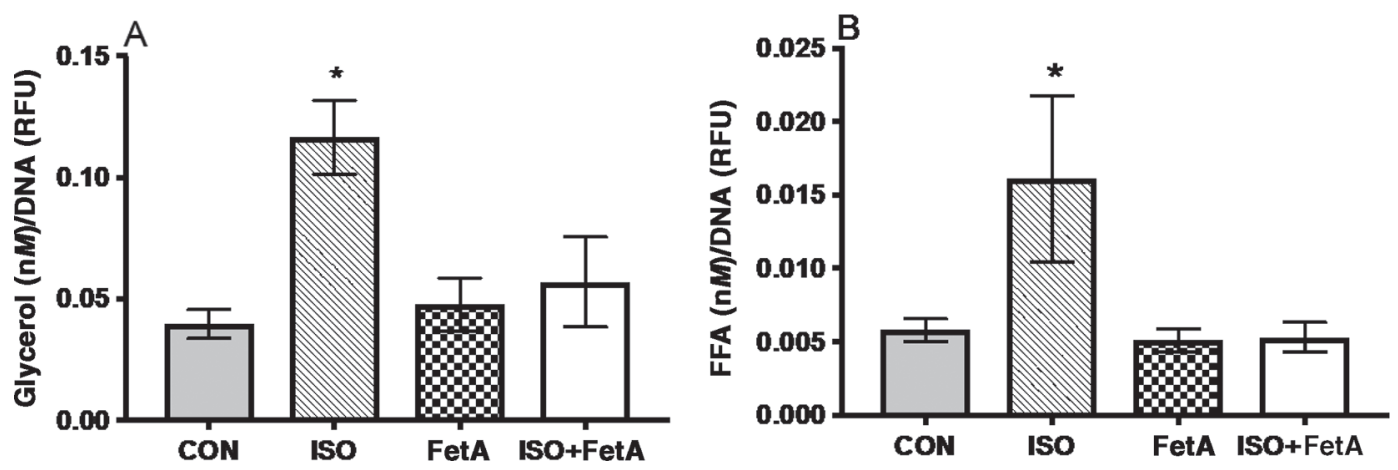

Figure 1. Effect of fetuin-A (FetA) on $\beta$-adrenergic induced lipolysis in bovine adipocytes $(\mathrm{n}=6)$. Concentrations of $(\mathrm{A})$ glycerol and $(\mathrm{B})$ free fatty acids (FFA) released in culture medium after a 2-h lipolysis induction with $1 \mu M$ isoproterenol (ISO). Concentrations of glycerol $(\mathrm{n} M)$ and FFA (nM) were standardized by the cellular DNA content [DNA relative fluorescence units (RFU)] measured by CyQUANT (Life Technologies, Carlsbad, CA). Values are showed as mean \pm SEM. ${ }^{*}$ Statistically different $(P<0.05)$. CON $=$ control, no reagents added.

\section{Fetuin-A Enhances Lipogenesis in Bovine Adipocytes}

Lipolysis and lipogenesis are continuous processes occurring simultaneously within adipocytes. Thus, we evaluated the effects of FetA on both lipolytic and lipogenic activity. We assessed lipogenesis by quantifying adipocyte FA uptake and accumulation of intracellular TAG. The FetA exposure during $1.5 \mathrm{~h}(0.5 \mathrm{~h}$ preincubation and 1-h kinetic assay) increased FA uptake by 1.5 times compared with $\mathrm{CON}(P=0.02$; Figure 2$)$. The effect of FetA on FA uptake was similar to that of insulin $(P=0.65$; Figure 2B), increasing FA uptake by 1.7 times compared with CON $(P=0.004$; Figure $2 \mathrm{~B})$. In agreement with the higher incorporation of FA, we observed that adipocytes treated with FetA for $48 \mathrm{~h}$ increased lipogenic capacity compared with $\mathrm{CON}(P<$ 0.05). Adipocytes in the FetA group had a higher accumulation of TAG as quantified with the neutral lipid fluorescent stain HCS LipidTOX $(P=0.003$; Figure $3 \mathrm{~A}-3 \mathrm{C}$ ) and with the quantitative AdipoRed assay ( $P$ $=0.04 ;$ Figure $3 \mathrm{D}-3 \mathrm{E})$.

\section{Fetuin-A Upregulates AGPAT2 Expression and Activity in Bovine Adipocytes}

To explore potential mechanisms by which FetA enhances lipogenic function in bovine adipocytes, we evaluated the effect of the adipokine on key regulators of adipogenesis (CEBPB, ADIPOQ, and PPARG), FA uptake $(F A B P 4, F A T P 1$, and FAT/CD36), and lipogenic enzymes controlling de novo TAG synthesis in adipocytes, such as AGPAT2, DGAT1, DGAT2, GPAT1, GPAT2, and LPIN1. We observed that FetA increased the transcription of AGPAT2 ( $P=0.02$; Figure $4 \mathrm{~A})$ and $A H S G(P=0.05$; Figure $4 \mathrm{~B})$ and tended to increase the expression of $C E B P B(P=0.07$; Figure $4 \mathrm{C})$ and FATP1 $(P=0.07$; Figure $4 \mathrm{D})$. No differ-
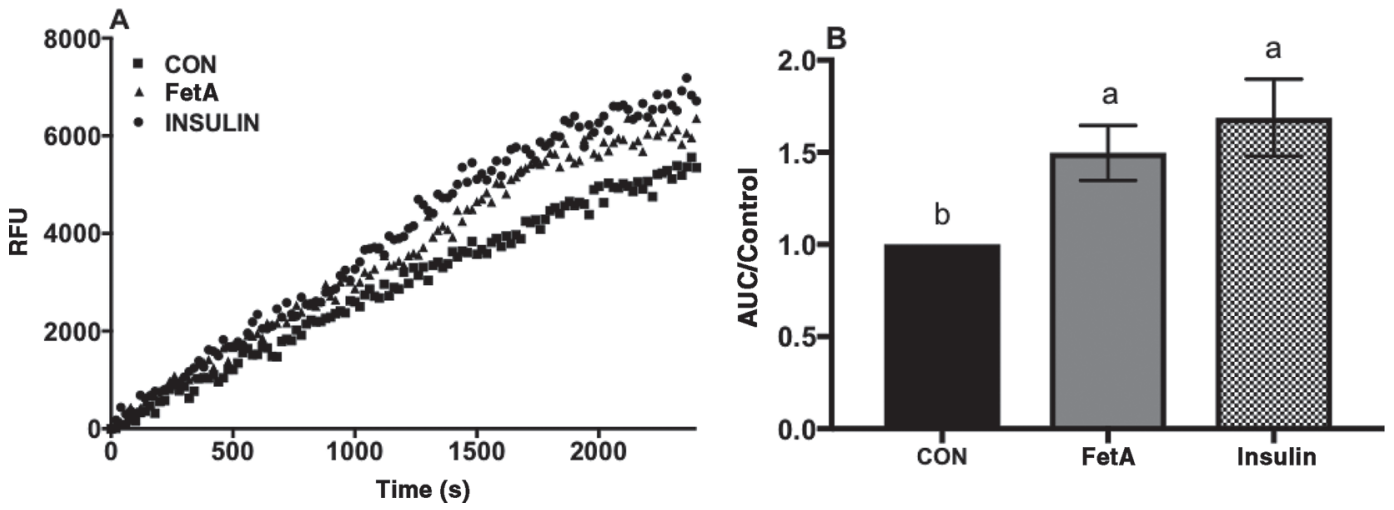

Figure 2. Effect of fetuin-A (FetA) on fatty acid (FA) uptake by bovine adipocytes $(\mathrm{n}=6)$. (A) Example of the kinetic QBT FA uptake (Molecular Devices, Sunnyvale, CA) output from bovine adipocytes over 1-h assay. RFU = relative fluorescence units. (B) Area under the curve (AUC) from FA uptake assays of bovine adipocytes treated with 0 (CON) or $0.1 \mathrm{mg} / \mathrm{mL}$ of FetA (FetA) or $10 \mathrm{n} M$ insulin (insulin). Bovine adipocytes were treated and pre-incubated with treatments for $30 \mathrm{~min}$ at $37^{\circ} \mathrm{C}$ before the assay. Values are expressed as AUC using CON FA uptake as the calibrator (means \pm SEM). Bars with different letters $(a, b)$ are significantly different $(P \leq 0.05)$. 
ence was observed in the gene expression of $P P A R G$, ADIPOQ, DGAT1, DGAT2, GPAT1, GPAT2, LPIN1, $F A T / C D 36$, and FABP 4 between FetA and CON $(P>$ 0.05). In agreement with the increase in AGPAT2 gene expression, AGPAT2 protein content was also increased by FetA compared with CON $(P=0.02$; Figure $4 \mathrm{~B})$.
The enzymatic activity of AGPAT2 during TAG synthesis transfers a FA to the lysophosphatidic acid molecule to produce PA. We assessed the effect of FetA on AGPAT2 activity by quantifying the concentrations of PA in bovine adipocytes treated with $0.1 \mathrm{mg} / \mathrm{mL}$ of FetA for 48 h. Adipocytes treated with FetA syn-
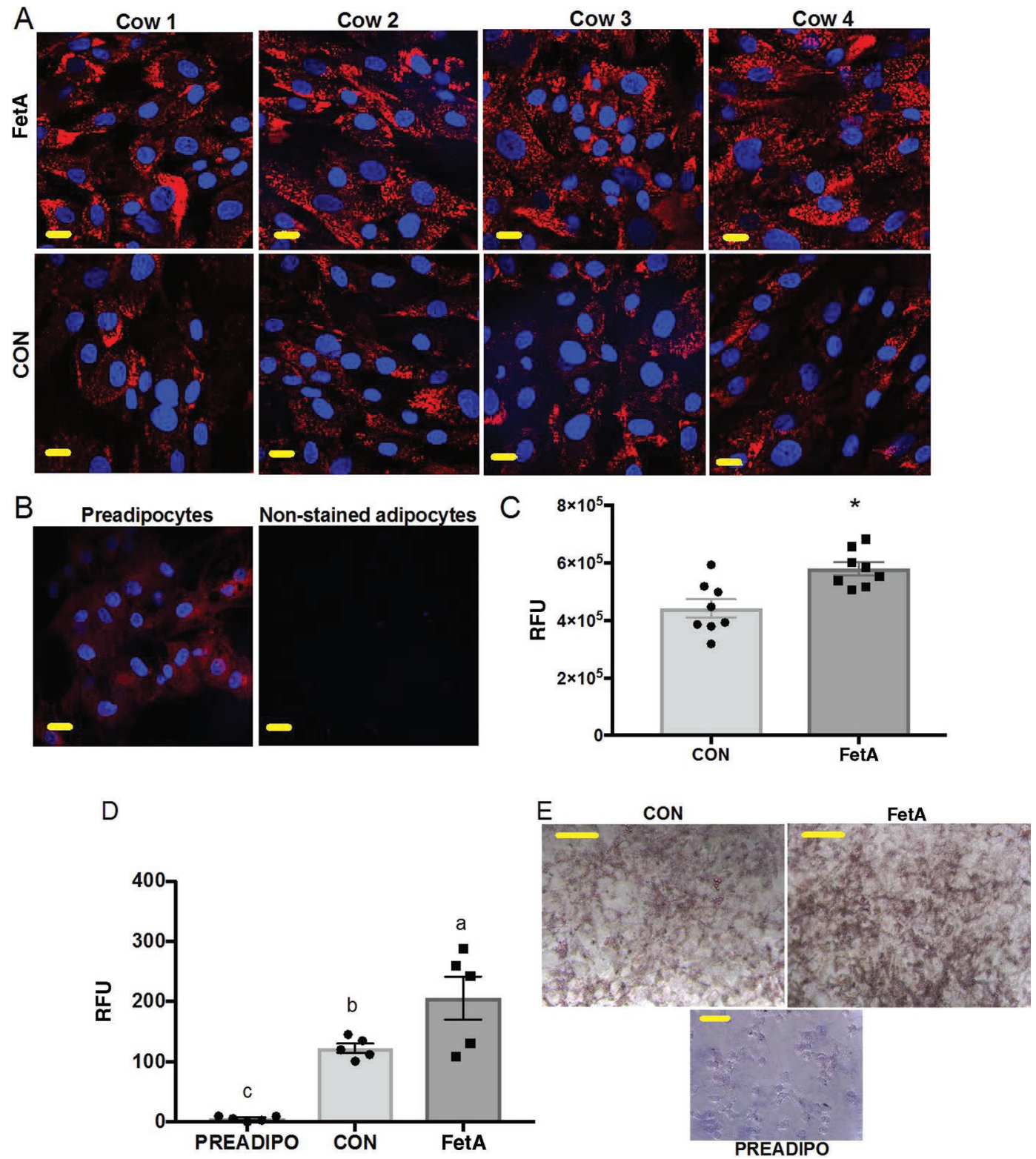

Figure 3. Effect of fetuin-A (FetA) on triacylglycerol accumulation in bovine adipocytes. (A) Laser scanning confocal microscopy imaging $(60 \times)$ of lipid droplets in cultured bovine adipocytes $(\mathrm{n}=8)$ treated with 0 (CON; bottom panel) or $0.1 \mathrm{mg} / \mathrm{mL}$ of FetA (FetA; upper panel). Scale bars: $20 \mu \mathrm{m}$. (B) Preadipocytes and nonstained cells served as controls. Lipid droplets of bovine adipocytes were stained with Alexa Fluor 594 (HCS LipidTox, Life Technologies, Carlsbad, CA; red fluorescence) and nuclei were stained with DAPI (NucBlue, Life Technologies; blue fluorescence). Scale bars: $20 \mu \mathrm{m}$. (C) Plotted relative fluorescence units (RFU) measured through ImageJ (https://imagej.net/Welcome) software (means \pm SEM) and calibrated by number of cells per image. ${ }^{*} P \leq 0.05$. (D) Relative fluorescent units of AdipoRed (Lonza, Allendale, $\mathrm{NJ}$ ) assay analysis in cultured bovine adipocytes $(\mathrm{n}=5)$ treated with $0(\mathrm{CON})$ or $0.1 \mathrm{mg} / \mathrm{mL}$ of FetA (FetA), and respective preadipocytes (PREADIPO) on $\mathrm{d}$ 0. Bars with different letters $(\mathrm{a}-\mathrm{c})$ are significantly different $(P \leq 0.05)$. (E) Representative microscopy images $(20 \times)$ of lipid droplets (dark red dots) in bovine adipocytes treated with $0(\mathrm{CON})$ or $0.1 \mathrm{mg} / \mathrm{mL}$ of FetA (FetA), and preadipocytes (PREADIPO) assayed with AdipoRed. Scale bars: $100 \mu \mathrm{m}$. 

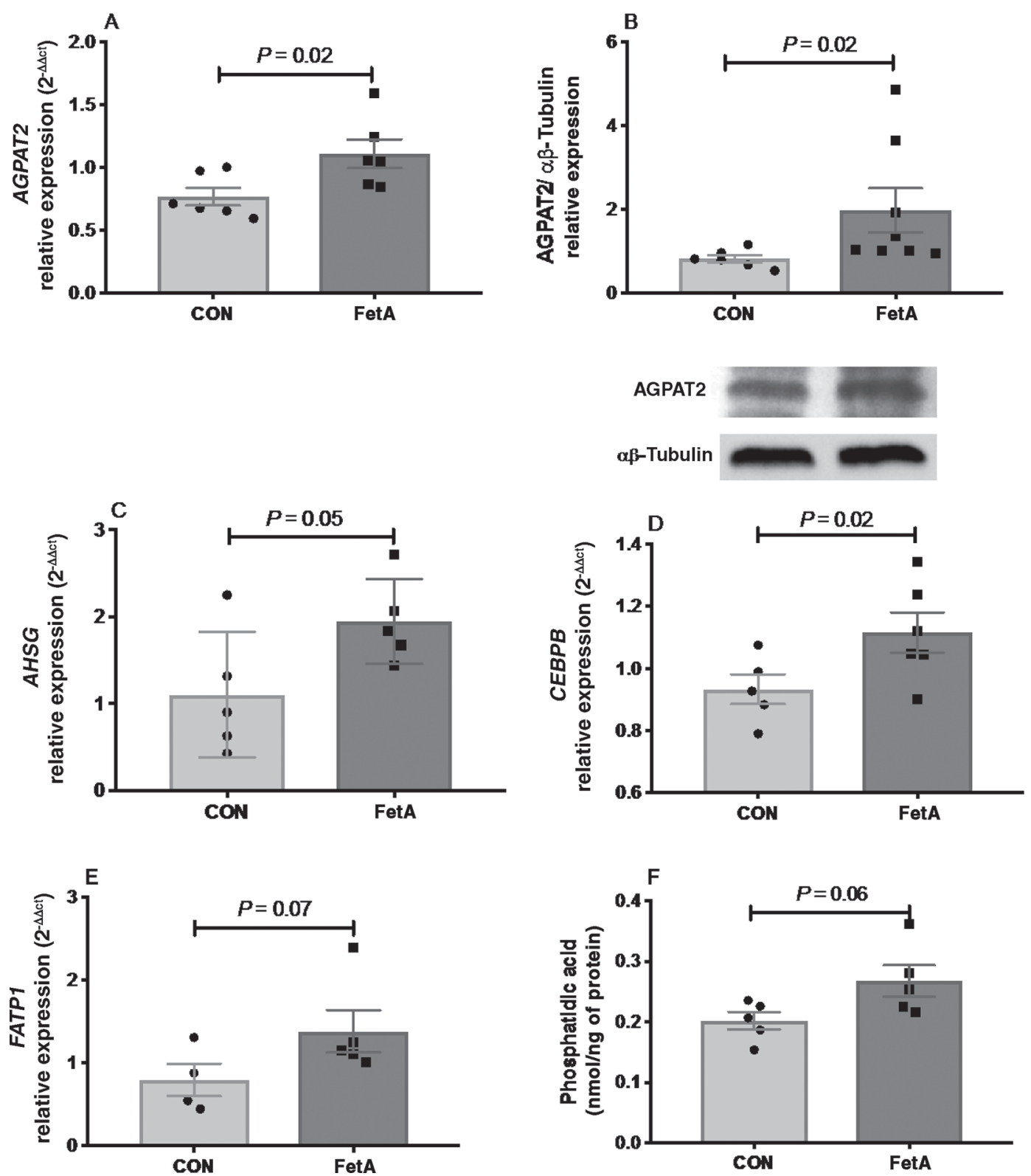

Figure 4. Effect of fetuin-A (FetA) on the expression of lipogenic markers and phosphatidic acid concentrations in bovine adipocytes. Adipocytes were treated with $0(\mathrm{CON})$ or $0.1 \mathrm{mg} / \mathrm{mL}$ of FetA (FetA) for $48 \mathrm{~h}$. (A) Gene expression and (B) protein content of 1-acylglycerol3-phosphate acyltransferase-2 (AGPAT2). Gene expression of (C) FetA (AHSG), (D) adipogenesis promoter CCAAT/enhancer-binding protein $\beta(C E B P B)$, and (E) fatty acid transporter-1 (FATP1). (F) Phosphatidic acid concentrations were calibrated by total protein concentrations (nmol/ng of protein). For quantitative PCR results, values were shown as relative gene expression $2^{(-\Delta \Delta \mathrm{CT})}$ (where CT $=\mathrm{cycle}$ threshold), normalized by control genes RPS9 and EIF3K, and represent mean \pm SEM. For Western blot analysis, $\alpha \beta$-tubulin was used as a loading control, and values are expressed as a ratio between FetA and $\alpha \beta$-tubulin adjusted band intensity $\left(\mathrm{mm}^{2}\right)$. Results represent means \pm SEM.

thetized more PA $(0.27 \pm 0.002 \mathrm{nmol} / \mathrm{ng}$ of protein $)$ compared with CON adipocytes $(0.20 \pm 0.01 \mathrm{nmol} / \mathrm{ng}$ of protein; $P=0.060$; Figure $4 \mathrm{~F})$.

\section{DISCUSSION}

The balance between lipogenesis and lipolysis in AT of periparturient dairy cows is critical to ensure a healthy and successful transition to lactation. Thus, reducing lipolysis or promoting lipogenesis are important therapeutic alternatives to improve periparturient cows' health. In our previous study, we observed that the expression of FetA and other lipogenic markers was downregulated in subcutaneous AT after parturition (Strieder-Barboza et al., 2018). Also, previous studies in nonruminants have consistently demonstrated the 
strong lipogenic properties of FetA (Cayatte et al., 1990; Pérez-Sotelo et al., 2017). To date, however, the mechanisms by which FetA increases TAG synthesis in adipocytes have not been characterized. In this study, we report for the first time that FetA modulates lipid mobilization in isolated and cultured bovine adipocytes and identify AGPAT2 activity as the potential mechanism for the lipogenic responses induced by FetA.

Previous studies demonstrate abundant mRNA and protein expression of FetA in AT of periparturient, mid-, and late-lactation dairy cows, as well as in cultured bovine adipocytes (Zachut et al., 2017; StriederBarboza et al., 2018). However, none of these studies had identified the source of FetA within AT. Here we demonstrate that primary adipocytes from subcutaneous AT of dairy cows not only express but also secrete FetA. Even though secretome studies of rodents' visceral and gonadal AT also detected significant amounts of FetA (Roca-Rivada et al., 2011), this is the first report of FetA secretion by bovine adipocytes. The synthesis of FetA is dynamic and depends on the metabolic status. In humans and rodents, FetA secretion decreased during fasting, weight loss, and anorexia and increased during weight gain and obesity (Jialal et al., 2015; Pérez-Sotelo et al., 2017). In dairy cows, serum concentrations and AT gene and protein expression of FetA decreased during early lactation and coincided with negative energy balance state (Strieder-Barboza et al., 2018). The changes in the FetA expression and secretion patterns in the AT of rodents, bovines, and humans during lipid mobilization suggest a role for FetA in the modulation of lipogenesis and lipolysis in adipocytes.

In the present study, FetA attenuated lipolytic responses induced by $\beta$-adrenergic stimulation (ISO). Our results suggest that the effect of FetA during inducedlipolysis in bovine adipocytes was insulin-independent because no insulin was added to the medium before and during $\beta$-adrenergic stimulation. Previous studies indicate that FetA may control insulin signaling in $\mathrm{AT}$ and is an attractive candidate gene for disturbed adipocyte lipolytic function in obesity and insulin resistance in humans (Dahlman et al., 2004). Adipose tissue lipolytic responsiveness and sensitivity to adrenergic agents, including catecholamines, are increased in dairy cows around parturition (Bell, 1995). Lipolysis increases the concentrations of FFA in plasma. Because high lipolytic rates are considered a risk factor for the development of diseases during the periparturient period (Grummer, 1993), attenuating lipolysis or regulating insulin function (or both) in early lactation would be particularly beneficial to dairy cows that develop hypoinsulinemia concurrently with a state of insulin resistance (Contreras et al., 2017b). Even though FetA seems to be involved in the pathways that control lipolytic responses in bovine adipocytes, the mechanisms by which this adipokine may play anti-lipolytic roles in dairy cows remain to be established. Additionally, since lipolysis and lipogenesis occur simultaneously within adipocytes, these results may also suggest that the responses observed could be directly related to enhanced lipogenesis.

In the present study, we observed that incubation of bovine adipocytes with FetA increased FA uptake compared with nontreated cells and induced similar responses to those observed in cells treated with the lipogenic hormone insulin. Although speculative at this time, FetA could have stimulated the translocation of plasma membrane FA transporters (i.e., FATP1 and CD36) similar to the known effects of insulin on FA trafficking. Another possibility is that FetA could have bound FA and translocated them into the intracellular compartment. Fatty acid transporters, such as albumin and FetA, are known for carrying lipids in plasma and facilitating FA uptake by cells (Glatz et al., 2010). Previously, FetA was reported to translocate into the cytoplasm of 3T3-L1 and human adipocytes (Dasgupta et al., 2010) and to accumulate in vesicles in the cytosol (Reynolds et al., 2005); nevertheless, its relationship with FA transport was not reported in these studies. Fetuin-A carries high amounts of cholesterol, cholesteryl esters, TAG, and FA in plasma, which correspond to almost $33 \%$ of its molecule (Kumbla et al., 1989, 1991). In rabbit and human cells, FetA increased the incorporation of exogenous FA into intracellular TAG by almost 50-fold compared with albumin (Cayatte et al., 1990). Similarly, we observed that FetA increases TAG accumulation in cultured bovine adipocytes compared with nontreated cells. This suggests that increased FetA-stimulated FA uptake by adipocytes might have enhanced the intracellular synthesis of TAG. Due to its lipogenic properties, increased serum concentrations and AT expression and secretion of FetA have been associated with obesity and obesity-related disorders in humans and animals (Chen et al., 2009; Jialal et al., 2015; Pérez-Sotelo et al., 2017). In dairy cows, we previously reported a decrease in serum FetA concentrations and its expression in AT from 2 wk prepartum to 10 DIM (Strieder-Barboza et al., 2018). This period of decreased FetA coincided with the downregulation in the transcription of lipogenic enzymes in the subcutaneous AT and with a marked increase of serum FFA concentrations in periparturient cows. Evidence from global gene expression profiling studies revealed that $A H S G$ might be involved in regulating energy metabolism in dairy cattle (Chen et al., 2011), and in lipid accumulation in several AT depots in beef cattle (Robinson and Oddy, 2004). Together, these results provide evidence 
that FetA may promote lipogenesis in vitro and in vivo in the AT of cows.

Although it is clear that FetA promotes the synthesis of TAG, the mechanisms involved are yet to be studied. Whether the stimulation of TAG accumulation in bovine adipocytes is due to the entry of FA into cells or whether FetA might directly stimulate lipogenic enzymes is not known. To address these questions, we evaluated the gene expression of key enzymes involved in the TAG synthesis through the glycerol-3-phosphate pathway in adipocytes: (1) GPAT, which is involved in glycerol-P synthesis; (2) AGPAT, a catalyst for the first step in the formation of PA; (3) phosphatidate phosphatases (lipin), which form diacylglycerol, and DGAT (Coleman and Lee, 2004; Takeuchi and Reue, 2009). In our study, FetA upregulated the expression of AGPAT2 and increased AGPAT2 protein content in adipocytes. The AGPAT2 is the predominant AGPAT isoform in AT and catalyzes acylation of its strict substrate lysophosphatidic acid to PA (Takeuchi and Reue, 2009). In cows, AGPAT play an important role in the TAG synthesis in the mammary gland (Mistry and Medrano, 2002), but have not yet been reported in AT. Peroxisome proliferator-activated receptor $\gamma$ agonists induce AGPAT2 activity (Blanchard et al., 2016) that it is required for TAG accumulation in mature adipocytes (Gale et al., 2006). Knocking down AGPAT2 decreased gene expression of adipogenesis regulators such as PPAR $\gamma$ and $C / E B P B$. Also, the absence of AGPAT2 delayed the expression of mature adipocyte markers such as FABP 4 and GLUT4, and reduced TAG accumulation in adipocytes (Blanchard et al., 2016). The crucial function of AGPAT2 for adipogenesis is highlighted by the near complete absence of AT and a range of metabolic changes, such as extreme insulin resistance, in humans with congenital generalized lipodystrophy as a consequence of AGPAT2 deficiency (Agarwal and Garg, 2006). These results demonstrate that other AGAPT family members (AGAPT1 and AGAPT3-10) cannot compensate for AGPAT2 activity, thus being a specific and rate-limiting enzyme for TAG synthesis (Agarwal et al., 2002; Takeuchi and Reue, 2009). Although we demonstrate that FetA enhances AGPAT2 activity, future studies are needed to evaluate if this activation is a consequence of increased FA uptake mediated by FetA or a direct signaling effect.

The AGPAT2 controls adipogenesis through modulation of the synthesis of phospholipids and TAG precursors, especially PA (Gale et al., 2006). Impaired AGPAT2 activity affects the availability of PA for TAG synthesis but not overall PA synthesis nor utilization of PA for phospholipid synthesis (Gale et al., 2006). In our study, we evaluated the effect of FetA on AGPAT2 activity by measuring adipocyte concentrations of PA. We observed that FetA increased not only gene expression and protein content of AGPAT2 in bovine adipocytes, but also PA concentrations. Phosphatidic acid was highlighted as one of the main lipid regulators of lipid droplet size, an important lipid-storage organelle (Fei et al., 2011). Our results suggest that FetA increases AGPAT2 activity, and therefore PA availability for TAG synthesis in bovine adipocytes. Knowing that FetA may modulate TAG synthesis precursors in adipocytes provides valuable insights into potential targets for modulating the flux of lipids in that AT of dairy cows.

It is important to mention that we analyzed the effect of FetA on a limited pool of genes involved in adipogenesis, FA uptake, and lipogenesis. Although we identified an effect of FetA on a few of these selected genes, it is possible that other lipogenic markers could be driving the lipogenic effect of FetA. Future studies using genetic manipulation of AGPAT2 and $A H S G$ expression in AT are needed to conclusively affirm that AGPAT2 signaling is required for the lipogenic effect of FetA in bovine AT.

\section{CONCLUSIONS}

Results from this study demonstrate that FetA modulates lipid mobilization by attenuating lipolytic responses and enhancing lipogenesis in bovine adipocytes. Fetuin-A upregulates the expression and activity of AGPAT2, a rate-limiting lipogenic enzyme, and suggests a potential mechanism by which this adipokine promotes TAG synthesis in adipocytes. Our study provides novel knowledge on how FetA promotes TAG synthesis in bovine adipocytes and opens the possibility of using FetA as a potential therapeutic target for the modulation of lipid mobilization in AT of periparturient dairy cows and humans with similar AT disorders.

\section{ACKNOWLEDGMENTS}

This research was supported by USDA-National Institute of Food and Agriculture (Washington, DC) grant 2015-67015-23207, Department of Large Animal Clinical Sciences (East Lansing, MI), Graduate School Dissertation Completion Fellowship-Spring 2018 (The Graduate School, Michigan State University), Michigan Alliance for Animal Agriculture (award AA18-003), and Global Agri-Trade Corporation (Gardena, CA). The authors are grateful to Kyan Thelen, Jenne De Koster, and Connor Lewicki; the staff at the Michigan State University Meat Laboratory (East Lansing) and West Michigan Beef (Hudsonville, MI) for providing samples; and Jeff Gandy (Department of Large Animal Clinical 
Sciences, Michigan State University, East Lansing) for technical assistance.

\section{REFERENCES}

Agarwal, A. K., E. Arioglu, S. de Almeida, N. Akkoc, S. I. Taylor, A. M. Bowcock, R. I. Barnes, and A. Garg. 2002. AGPAT2 is mutated in congenital generalized lipodystrophy linked to chromosome 9q34. Nat. Genet. 31:21-23.

Agarwal, A. K., and A. Garg. 2006. Genetic basis of lipodystrophies and management of metabolic complications. Annu. Rev. Med. $57: 297-311$.

Bauman, D. E., and W. B. Currie. 1980. Partitioning of nutrients during pregnancy and lactation: A review of mechanisms involving homeostasis and homeorhesis. J. Dairy Sci. 63:1514-1529.

Bell, A. W. 1995. Regulation of organic nutrient metabolism during transition from late pregnancy to early lactation. J. Anim. Sci. 73:2804-2819.

Blanchard, P. G., V. Turcotte, M. Côté, Y. Gélinas, S. Nilsson, G. Olivecrona, Y. Deshaies, and W. T. Festuccia. 2016. Peroxisome proliferator-activated receptor $\gamma$ activation favours selective subcutaneous lipid deposition by coordinately regulating lipoprotein lipase modulators, fatty acid transporters and lipogenic enzymes. Acta Physiol. (Oxf.) 217:227-239.

Cayatte, A. J., L. Kumbla, and M. T. Subbiah. 1990. Marked acceleration of exogenous fatty acid incorporation into cellular triglycerides by fetuin. J. Biol. Chem. 265:5883-5888.

Chen, H.-Y., Y.-L. Chiu, S.-P. Hsu, M.-F. Pai, C.-F. Lai, Y.-S. Peng, T.-W. Kao, K.-Y. Hung, T.-J. Tsai, and K.-D. Wu. 2009. Association of serum fetuin A with truncal obesity and dyslipidemia in non-diabetic hemodialysis patients. Eur. J. Endocrinol. 160:777783.

Chen, Y., C. Gondro, K. Quinn, R. Herd, P. Parnell, and B. Vanselow. 2011. Global gene expression profiling reveals genes expressed differentially in cattle with high and low residual feed intake. Anim. Genet. 42:475-490.

Coleman, R. A., and D. P. Lee. 2004. Enzymes of triacylglycerol synthesis and their regulation. Prog. Lipid Res. 43:134-176.

Contreras, G. A., C. Strieder-Barboza, and J. De Koster. 2018. Symposium review: Modulating adipose tissue lipolysis and remodeling to improve immune function during the transition period and early lactation of dairy cows. J. Dairy Sci. 101:2737-2752.

Contreras, G. A., C. Strieder-Barboza, J. de Souza, J. Gandy, V. Mavangira, A. L. Lock, and L. M. Sordillo. 2017a. Periparturient lipolysis and oxylipid biosynthesis in bovine adipose tissues. PLoS One 12:e0188621.

Contreras, G. A., C. Strieder-Barboza, and W. Raphael. 2017b. Adipose tissue lipolysis and remodeling during the transition period of dairy cows. J. Anim. Sci. Biotechnol. 8:41.

Dahlman, I., P. Eriksson, M. Kaaman, H. Jiao, C. Lindgren, J. Kere, and P. Arner. 2004. a2-Heremans-Schmid glycoprotein gene polymorphisms are associated with adipocyte insulin action. Diabetologia 47:1974-1979.

Dasgupta, S., S. Bhattacharya, A. Biswas, S. S. Majumdar, S. Mukhopadhyay, and S. Ray. 2010. NF-kappaB mediates lipid-induced fetuin-A expression in hepatocytes that impairs adipocyte function effecting insulin resistance. Biochem. J. 429:451-462.

Fei, W., G. Shui, Y. Zhang, N. Krahmer, C. Ferguson, T. S. Kapterian, R. C. Lin, I. W. Dawes, A. J. Brown, and P. Li. 2011. A role for phosphatidic acid in the formation of "supersized" lipid droplets. PLoS Genet. 7:e1002201.

Gale, S. E., A. Frolov, X. Han, P. E. Bickel, L. Cao, A. Bowcock, J. E. Schaffer, and D. S. Ory. 2006. A regulatory role for 1-acylglycerol3-phosphate-O-acyltransferase 2 in adipocyte differentiation. J. Biol. Chem. 281:11082-11089.
Glatz, J. F., J. J. Luiken, and A. Bonen. 2010. Membrane fatty acid transporters as regulators of lipid metabolism: Implications for metabolic disease. Physiol. Rev. 90:367-417.

Grummer, R. R. 1993. Etiology of lipid-related metabolic disorders in periparturient dairy cows. J. Dairy Sci. 76:3882-3896.

Heinrichsdorff, J., and J. M. Olefsky. 2012. Fetuin-A: The missing link in lipid-induced inflammation. Nat. Med. 18:1182-1183.

Hellemans, J., G. Mortier, A. De Paepe, F. Speleman, and J. Vandesompele. 2007. qBase relative quantification framework and software for management and automated analysis of real-time quantitative PCR data. Genome Biol. 8:R19.

Jialal, I., S. Devaraj, A. Bettaieb, F. Haj, and B. Adams-Huet. 2015. Increased adipose tissue secretion of Fetuin-A, lipopolysaccharidebinding protein and high-mobility group box protein 1 in metabolic syndrome. Atherosclerosis 241:130-137.

Kumbla, L., S. Bhadra, and M. T. Subbiah. 1991. Multifunctional role for fetuin (fetal protein) in lipid transport. FASEB J. 5:2971-2975.

Kumbla, L., A. Cayatte, and M. Subbiah. 1989. Association of a lipoprotein-like particle with bovine fetuin. FASEB J. 3:2075-2080.

McNamara, J. P. 1995. Role and regulation of metabolism in adipose tissue during lactation. J. Nutr. Biochem. 6:120-129.

Mistry, D. H., and J. F. Medrano. 2002. Cloning and localization of the bovine and ovine lysophosphatidic acid acyltransferase (LPAAT) genes that codes for an enzyme involved in triglyceride biosynthesis. J. Dairy Sci. 85:28-35.

Pal, D., S. Dasgupta, R. Kundu, S. Maitra, G. Das, S. Mukhopadhyay, S. Ray, S. S. Majumdar, and S. Bhattacharya. 2012. Fetuin-A acts as an endogenous ligand of TLR4 to promote lipid-induced insulin resistance. Nat. Med. 18:1279-1285.

Pérez-Sotelo, D., A. Roca-Rivada, M. Larrosa-García, C. Castelao, I. Baamonde, J. Baltar, A. B. Crujeiras, L. M. Seoane, F. F. Casanueva, and M. Pardo. 2017. Visceral and subcutaneous adipose tissue express and secrete functional alpha2hsglycoprotein (fetuin A) especially in obesity. Endocrine 55:435-446.

Reynolds, J. L., J. N. Skepper, R. McNair, T. Kasama, K. Gupta, P. L. Weissberg, W. Jahnen-Dechent, and C. M. Shanahan. 2005. Multifunctional roles for serum protein fetuin-A in inhibition of human vascular smooth muscle cell calcification. J. Am. Soc. Nephrol. 16:2920-2930

Robinson, D., and V. Oddy. 2004. Genetic parameters for feed efficiency, fatness, muscle area and feeding behaviour of feedlot finished beef cattle. Livest. Prod. Sci. 90:255-270.

Roca-Rivada, A., J. Alonso, O. Al-Massadi, C. Castelao, J. R. Peinado, L. M. Seoane, F. F. Casanueva, and M. Pardo. 2011. Secretome analysis of rat adipose tissues shows location-specific roles for each depot type. J. Proteomics 74:1068-1079.

Strieder-Barboza, C., J. de Souza, W. Raphael, A. L. Lock, and G. A. Contreras. 2018. Fetuin-A: A negative acute-phase protein linked to adipose tissue function in periparturient dairy cows. J. Dairy Sci. 101:2602-2616.

Takeuchi, K., and K. Reue. 2009. Biochemistry, physiology, and genetics of GPAT, AGPAT, and lipin enzymes in triglyceride synthesis. Am. J. Physiol. Endocrinol. Metab. 296:E1195-E1209.

Thelen, K., S. W. Watts, and G. A. Contreras. 2018. Adipogenic potential of perivascular adipose tissue preadipocytes is improved by coculture with primary adipocytes. Cytotechnology 70:1435-1445.

Vernon, R., and K. Houseknecht. 2000. Adipose tissue: Beyond an energy reserve. Pages 171-186 in Ruminant Physiology: Digestion, Metabolism, Growth and Reproduction. P. Cronjé, ed. CABI Publishing, New York, NY.

Vernon, R. G., and C. M. Pond. 1997. Adaptations of maternal adipose tissue to lactation. J. Mammary Gland Biol. Neoplasia 2:231-241.

Zachut, M., G. Kra, L. Livshitz, Y. Portnick, S. Yakoby, G. Friedlander, and Y. Levin. 2017. Seasonal heat stress affects adipose tissue proteome toward enrichment of the Nrf2-mediated oxidative stress response in late-pregnant dairy cows. J. Proteomics 158:52-61. 